\title{
Renormalization of Commensurate Magnetic Peak in Ni-doped $\mathrm{La}_{1.85} \mathrm{Sr}_{0.15} \mathrm{CuO}_{4}$
}

\author{
Masato Matsuura ${ }^{* \dagger}$, Maiko Kofu ${ }^{1}$, Hiroyuki Kimura ${ }^{1}$, and Kazuma Hirota* \\ Institute for Solid State Physics, University of Tokyo, Kashiwa 277-8581 \\ ${ }^{1}$ Institute of Multidisciplinary Research for Advanced Materials, Tohoku University, Sendai 980-8577
}

We have studied the magnetic excitations in impurity doped $\mathrm{La}_{1.85} \mathrm{Sr}_{0.15} \mathrm{Cu}_{1-y} \mathrm{~A}_{y} \mathrm{O}_{4}$ $(\mathrm{A}=\mathrm{Ni}$ or $\mathrm{Zn})$ by neutron scattering. The dispersion for $\mathrm{Zn}: y=0.017$ is similar to that for the impurity free sample: incommensurate peaks with the incommensurability $\delta=0.12 \pm 0.01$ (rlu) do not change their positions up to $21 \mathrm{meV}$. On the other hand, for Ni: $y=0.029$, two incommensurate peaks observed at low energies suddenly change into a broad commensurate peak at $E_{\text {cross }}=15 \mathrm{meV}$. Compared to the impurity free sample with a similar Sr-concentration $x=0.16, E_{\text {cross }}$ for Ni: $y=0.029$ is decreased by nearly the same factor for the reduction in $T_{c}$. This is very similar to the shift of the resonance energy $\left(E_{\mathrm{res}}\right)$ in Ni-doped $\mathrm{YBa}_{2} \mathrm{Cu}_{3} \mathrm{O}_{7}$. These common impurity effects on the shift of $E_{\text {cross }}$ and $E_{\text {res }}$ suggest the same magnetic origin for the resonance peak in $\mathrm{YBa}_{2} \mathrm{Cu}_{3} \mathrm{O}_{\delta}$ and that for a crossing point of upward and downward dispersions in the $\mathrm{La}_{2-x} \mathrm{Sr}_{x} \mathrm{CuO}_{4}$. We propose that the sudden change in the dispersion is better described by a crossover from incommensurate spin fluctuations to a gapped spin wave rather than a hourglass-like dispersion.

\section{KEYWORDS: high-temperature superconductor, LSCO, neutron scattering, impurity, magnetic excitation}

\section{Introduction}

Recently, similar magnetic excitations have been observed in high transition temperature (high- $T_{c}$ ) cuprates by inelastic neutron scattering (INS), namely, a characteristic hourglasslike magnetic excitation. ${ }^{1-7}$ The hourglass-like excitations consist of downward and upward dispersions from an antiferromagnetic (AF) zone center, the so-called $(\pi, \pi)$, and include two characteristic magnetic signals; low-energy incommensurate peaks and a resonance peak. The former signal has been observed in the two typical high- $T_{c}$ cuprates; $\mathrm{YBa}_{2} \mathrm{Cu}_{3} \mathrm{O}_{6+\delta}(\mathrm{YBCO})$ and $\mathrm{La}_{2-x} \mathrm{Sr}_{x} \mathrm{CuO}_{4}$ (LSCO), and it has been revealed that the incommensurability $\delta$ is scaled by $T_{c}$ in the underdoped and optimally-doped regions. ${ }^{8,9}$ The resonance peak is the most dominant magnetic excitation signal in bilayer cuprates, such as $\mathrm{YBCO}$ and $\mathrm{Bi}_{2} \mathrm{Sr}_{2} \mathrm{CaCu}_{2} \mathrm{O}_{8+\delta}$ (Bi2212). ${ }^{10,11}$ The resonance peak appears at $\mathbf{Q}_{\|}=(\pi, \pi)$ and $\omega \sim 40 \mathrm{meV}$, which corresponds to a crossing point of upward and downward dispersions in the hourglass-like dispersion. The

\footnotetext{
${ }^{*}$ Present address: Department of Earth and Space Science, Faculty of Science, Osaka University, Toyonaka, 560-0043.

${ }^{\dagger}$ E-mail address: mmatsuura@ess.sci.osaka-u.ac.jp
} 
resonance peak in the $\mathrm{YBCO}$ and $\mathrm{Bi} 2212$ systems grows below $T_{c}$ like an order-parameter, ${ }^{10}$ and the resonance energy $E_{\text {res }}$ is scaled by $T_{c}\left(5.8 k_{\mathrm{B}} T_{c}\right) .{ }^{9}$ Although the energy of the crossing point $\left(E_{\text {cross }}\right)$ in the LSCO system scales with $T_{c}\left(E_{\text {cross }} \sim 12 k_{\mathrm{B}} T_{c}\right)$ in the superconducting phase, ${ }^{7,12,13}$ the $(\pi, \pi)$-peak at the crossing point in the LSCO does not show any clear enhancement below $T_{c}{ }^{7}$ These experimental facts give rise to the following questions. Are the origin of the resonance peak in YBCO and that of the crossing point in LSCO same? Is there any universal mechanism to determine $E_{\text {res }}$ and $E_{\text {cross }}$ in high- $T_{c}$ cuprates?

Impurity effects on $\mathrm{Cu}$ sites, in particular, nonmagnetic $\mathrm{Zn}^{2+}(S=0)$ and magnetic $\mathrm{Ni}^{2+}$ $(S=1)$, have been used to study a correlation between the magnetism and the superconductivity because it can control $T_{c}$ without changing a carrier number and lattice properties. Therefore, studying impurity effects on $E_{\text {cross }}$ can separate magnetic and non-magnetic contributions on the $T_{c}$-scaling of $E_{\text {cross }}$. In addition, the impurity effects of $\mathrm{Ni}$ and $\mathrm{Zn}$ on $E_{\text {res }}$ in YBCO seem different: Zn does not change $E_{\text {res }}$ while Ni decreases $E_{\text {res }}$ with a preserved $E_{\text {res }} / T_{c}$ ratio. ${ }^{14}$ Comparing impurity effects on $E_{\text {cross }}$ in LSCO and $E_{\text {res }}$ in YBCO could give a clue to their origins. In this report, we present INS measurement on impurity doped $\mathrm{La}_{1.85} \mathrm{Sr}_{0.15} \mathrm{Cu}_{1-y} \mathrm{~A}_{y} \mathrm{O}_{4}(\mathrm{~A}=\mathrm{Ni}: y=0.029$ and $\mathrm{Zn}: y=0.017)$. We observed that $E_{\text {cross }}$ for Ni: $y=0.029$ is reduced by the same factor for the reduction of $T_{c}$, which is similar to the decrease in $E_{\text {res }}$ for the Ni-doped YBCO. ${ }^{14}$

\section{Experimental Deitals}

Single crystals were carefully grown using a traveling-solvent floating zone method with a special attention to homogeneity of the doped-impurity. The crystals used in this report are same as those used in ref. 15, and the detailed sample characterizations were described therein. $T_{c}$, defined at midpoint of transition, drops from $36.8 \mathrm{~K}(y=0)$ to $11.6 \mathrm{~K}(\mathrm{~A}=\mathrm{Ni}: y=$ $0.029)$ and to $16.0 \mathrm{~K}(\mathrm{~A}=\mathrm{Zn}: y=0.017)$, while the structural transition temperatures from tetragonal to orthorhombic phase are nearly same: $186(y=0), 182(\mathrm{Ni}: y=0.029)$, and $202 \mathrm{~K}$ $(\mathrm{Zn}: y=0.017)$, suggesting the same Sr-concentration for these samples. INS experiments were performed using the Tokyo University's triple axis spectrometer PONTA installed at the JRR3 reactor in the Japan Atomic Energy Agency. The horizontal collimations were $40^{\prime}-40^{\prime}-80^{\prime}-80^{\prime}$. The final neutron energy was fixed at $14.7 \mathrm{meV}$ with a pyrolytic graphite $(\mathrm{PG})$ analyzer. A PG filter was placed in front of the analyzer to diminish scattering for higher order neutrons. Throughout this paper, we label the momentum transfer $\left(Q_{x}, Q_{y}, Q_{z}\right)$ in units of reciprocal lattice vectors $a^{*} \sim b^{*}=1.178 \AA^{-1}$ and $c^{*}=0.4775 \AA^{-1}$ for the orthogonal notaion.

For the monolayer high- $T_{c}$ cuprates, magnetic scattering forms a rod-like signal along the $l$-direction in the $Q$-space due to the two-dimensional spin correlation in the real-space. We mounted the samples in the $(h, 0, l)$ ortho zone and chose $l$ to make the instrumental resolution ellipsoid parallel to the magnetic rod as shown in a schematic diagram in Fig.1(m) to get maximum magnetic signal. Such $l^{\prime}$ 's $\left(l_{\|}\right.$'s) are $-2.1,-2.2,-2.3,-2.6,-2.6,-2.9,-3.0$, and -3.3 for 
$\omega=12,13,14,15,16,17,18$, and $21 \mathrm{meV}$, respectively. In the three dimensional reciprocal space, four incommensurate peaks appear at $\left(\frac{1}{2} \pm \delta, \frac{1}{2}, 0\right)$ and $\left(\frac{1}{2}, \frac{1}{2} \pm \delta, 0\right)$ in the tetragonal notation, which correspond to $(1 \pm \delta, \delta, 0)$ and $(1 \pm \delta,-\delta, 0)$ in the orthorhombic notation. Although the incommensurate peaks are not in the $(h, 0, l)_{\text {ortho }}$ zone, signals are detectable owing to a broad $q$-resolution along $q_{z}$ ( $k_{\text {ortho }}$ in this case) direction as shown in a schematic diagram in Fig.1(n). In addition to the tune of the instrumental resolution, we averaged signals at several l's. Because a coherent single phonon scattering has a strong $l$-dependence while a magnetic signal has almost no $l$-dependence, this procedure dilutes phonon contributions and makes magnetic signals clearer. All the profiles of constant-energy scans in this report are average of five constant-energy scans along the $[100]_{\text {ortho }}$ direction around (10l). Figure 1 shows examples of such $l$-average procedure for the Ni: $y=0.029$ with energy transfer fixed at 12 and $15 \mathrm{meV}$. The fixed five $l_{\text {'s }}$ are selected around $l_{\|}$with \pm 0.2 and \pm 0.4 (rlu). Spurious peaks such as Figs. 1(a) $(1.2,0,-1.7)$ and (b) $(1.15,0,-1.9)$ were not used for this $l$-average procedure. Longitudinal acoustic and optical phonon branches along [110] tetra in $\mathrm{La}_{1.9} \mathrm{Sr}_{0.1} \mathrm{CuO}_{4}$ were reported around 14 and $18 \mathrm{meV}$ at the zone boundary, respectively. ${ }^{16}$ Although phonon or spurious peaks appear at some $l$ 's, similar peak structures, incommensurate peaks for $\omega=$ $12 \mathrm{meV}$ and a single broad peak for $\omega=15 \mathrm{meV}$, were commonly observed through five $l$ 's. These peak features are clearer for the summed profiles as shown in Figs. 1(f) and (l).

\section{Experimental Results}

Figures 2 (a)-(c) show profile of constant-energy scans at $\omega=12,15$, and $21 \mathrm{meV}$ obtained by the $l$-average procedure. For Zn: $y=0.017$, the incommensurate peaks appear at the same position, $h=1 \pm \delta$ with $\delta=0.12 \pm 0.01$ (rlu) for all energies, which is similar to the impurity free sample $y=0$. Two symmetrical Gaussians reproduce the data for Zn: $y=0.017$ well even at $21 \mathrm{meV}$, where optical phonon modes are expected, which confirms validity of the $l$-average procedure. For Ni: $y=0.029$, at $\omega=12 \mathrm{meV}$, the incommensurate peaks were observed at the same $\delta=0.116 \pm 0.007$ as $y=0$ and Zn: $y=0.017$. On the other hand, the two incommensurate peaks merge into a single broad peak for $\omega \geq 15 \mathrm{meV}$ of Ni: $y=0.029$. Because a drastic change in lattice dynamics is hardly expected by a small amount of impurities, the single broad peak above $\omega \geq 15 \mathrm{meV}$ for $\mathrm{Ni}: y=0.029$ cannot be explained by phonon contributions which is absent for Zn: $y=0.017$. Therefore, the changes in these profiles are associated with a change in magnetism and/or superconductivity by Ni-doping.

To explore the in-plane dispersion for Ni: $y=0.029$, constant-energy scans were carried out at several energies at $T=11 \mathrm{~K}$ as shown in Figs. 3. For $12 \leq \omega \leq 14 \mathrm{meV}$, the incommensurability $\delta$ varies little with increasing energy. At $\omega=15 \mathrm{meV}$, the scattering intensity around the AF zone center increases, and the two incommensurate peaks suddenly become a single broad peak. Above $16 \mathrm{meV}$, although background becomes high owing to optical phonons at 18 and $21 \mathrm{meV},{ }^{17}$ a broad peak was commonly observed at the AF zone center. 
J. Phys. Soc. Jpn.

Full PAPER
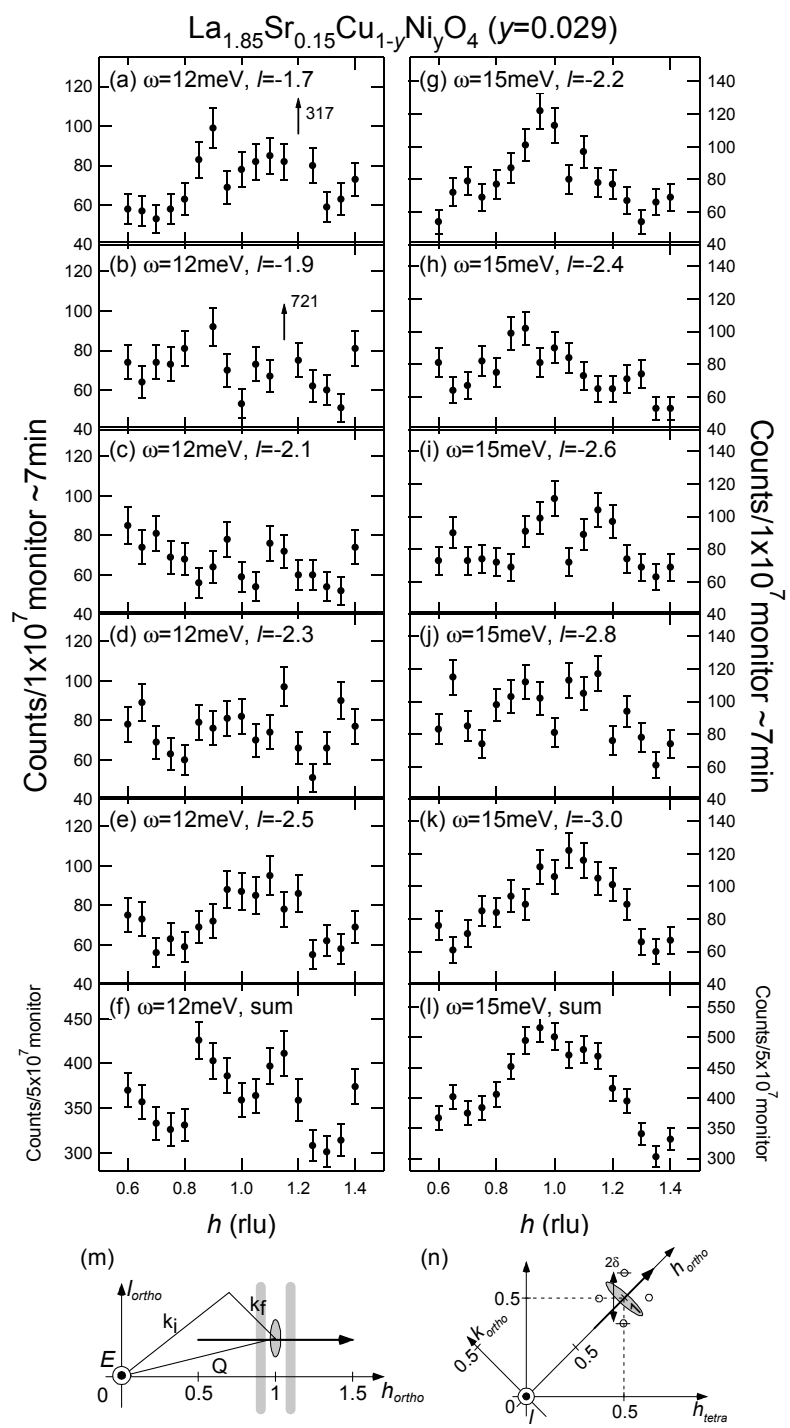

Fig. 1. Profiles of constant- $E$ scan of Ni: $y=0.029$ with energy transfer fixed at (a)-(e) $12 \mathrm{meV}$ and (g)-(k) $15 \mathrm{meV}$ measured at $T=11 \mathrm{~K}$. The trajectory of the scan is along [100] $]_{\text {ortho }}$ around the AF zone center $(10 l)$, which is shown by the arrow in $(\mathrm{m})$. To get a sharp peak, the instrumental resolution (gray ellipsoid) is tuned to be parallel to magnetic rod (two gray rods) by selecting $l$ of the scans. At each energy, five [100] ortho-scans at different fixed l's were measured and summed up to dilute phonon peaks and make magnetic signals clearer as described in the text. The summed profiles are shown in (f) and (l) for $\omega=12$ and $15 \mathrm{meV}$, respectively. (n) Wave vectors of the incommensurate peaks in the $(h k 0)$ zone in tetragonal and orthorhombic notations.

Figure 4 shows the same constant-energy scans measured at $T=300 \mathrm{~K}$. All profiles show monotonical decrease with increasing $h$ and have no appreciable peak structure. Therefore, the broad peak structure observed at $T=11 \mathrm{~K}$ above $\omega \geq 15 \mathrm{meV}$ disappears at high temperatures, indicating magnetic origin of these peaks. 


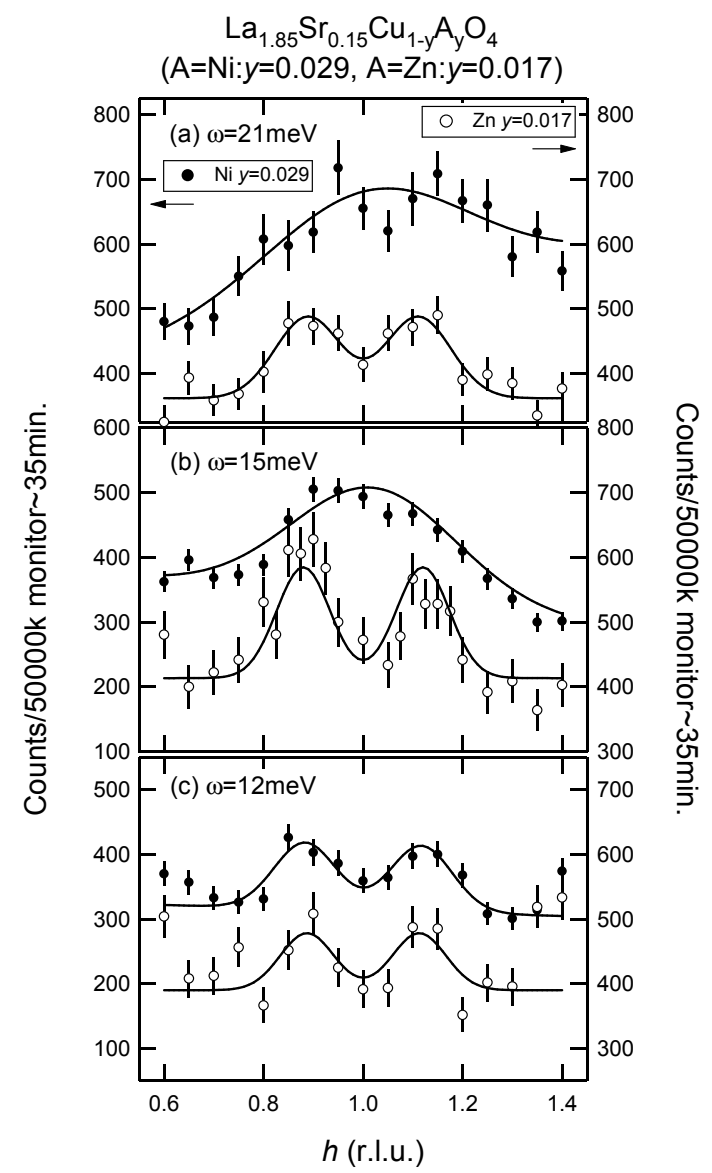

Fig. 2. (a)-(c) Profiles of constant- $E$ scan along the $[100]_{\text {ortho }}$ direction around the AF zone center $(h=1.0)$ at $T=11 \mathrm{~K}$ for Ni: $y=0.029$ (closed circles) and Zn: $y=0.017$ (solid circles).

\section{Discussion}

The $Q$-integrated susceptibility $\chi^{\prime \prime}(\omega)$ for Ni: $y=0.029$ and Zn: $y=0.017$ are shown in Fig. 5(a). $\chi^{\prime \prime}(\omega)$ for Ni:y $=0.029$ exhibits a maximum near $15-16 \mathrm{meV}$ at which the two incommensurate peaks merge into the broad commensurate peak at $(\pi, \pi)$. The maximum $\chi^{\prime \prime}(\omega)$ at $E_{\text {res }}$ or $E_{\text {cross }}$ is commonly observed in high- $T_{c}$ cuprates. Together with a change from the incommensurate peak structure to the commensurate one, we conclude that $E_{\text {cross }}$ for Ni: $y=0.029$ is $15-16 \mathrm{meV}$, which is 2.7 times smaller than $E_{\text {cross }}$ for the impurity free LSCO with similar Sr-concentration $x=0.16 .^{7}$ Hiraka et al. revealed that doped Ni-ions form a strong bound state with holes and reduce the effective hole carrier number, e.g. $x=0.15$ with $y=0.029$ corresponds to $x=0.121$ with $y=0 .{ }^{18}$ However, the reduction in $E_{\text {cross }}$ can not be explained only by reduction in the hole carrier, because $E_{\text {cross }}$ of $25 \mathrm{meV}$ for $x=0.10^{12}$ is even higher than the current result on Ni:y $=0.029$. Instead, $E_{\text {cross }}$ decreases with a preserved $E_{\text {cross }} / T_{c}$ ratio.

$\chi^{\prime \prime}(\omega)$ and dispersion for Zn: $y=0.017$ are shown in Figs. 5(a) and (b) as open circles. 


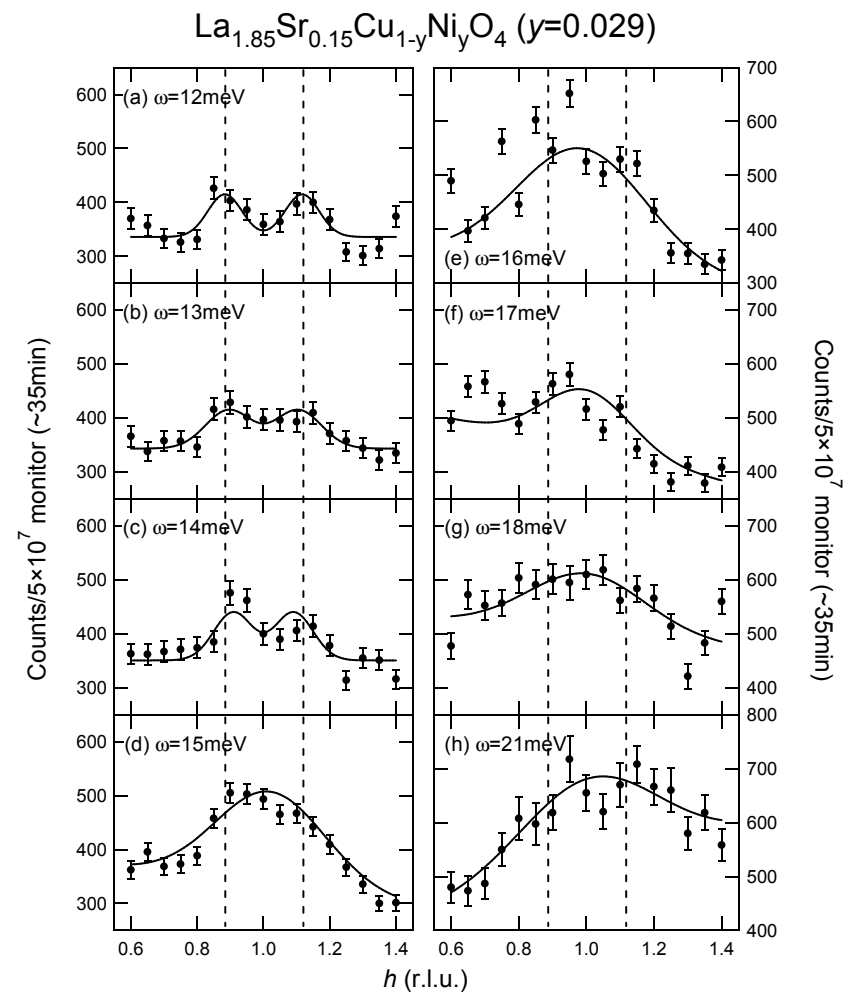

Fig. 3. (a)-(c) Profiles of $l$-averaged constant-energy scans at several energies for Ni: $y=0.029$ measured at $T=11 \mathrm{~K}$. The trajectory of the scan is same as Fig. 1. Solid lines show fit to two symmetrical Gaussian functions around $h=1.0$ for $\omega \leq 14 \mathrm{meV}$ and to a single Gaussian one at $h=1.0$ for $\omega \geq 15 \mathrm{meV}$ on a sloped background. The vertical broken lines show the positions of the incommensurate peaks at $\omega=12 \mathrm{meV}$.

The incommensurability $\delta$ is same as impurity free sample and is constant up to $21 \mathrm{meV}$, which indicates that Zn-doping does not affect $E_{\text {cross }}$. Sidis et al. have studied the impurity (Ni and $\mathrm{Zn}$ ) effect on $E_{\text {res }}$ in optimally doped YBCO, and found that the resonance peak shifts to lower energy by Ni-doping with a preserved $E_{\text {res }} / T_{c}$ ratio while Zn-doping does not change $E_{\text {res }} \cdot{ }^{14}$ These similar impurity effects on $E_{\text {cross }}$ and $E_{\text {res }}$ indicate a common origin for the crossing point peak in LSCO and the resonance peak in YBCO.

The marked difference between the LSCO and the YBCO families is the thermal variation of the magnetic spectrum at $E_{\text {cross }}$ or $E_{\text {res }}$; an order-parameter like behavior only for the resonance peak. However, for the $\mathrm{Zn}$-doped optimum YBCO, the intensity of the resonance peak gradually decreases with increasing temperature, and a substantial spectral weight remains above $T_{c} \cdot{ }^{14}$ Recently, Koike $e l$ al. have revealed a small superconducting volume fraction in a wide doping range of LSCO due to phase separation and/or spatial inhomogeneity. ${ }^{19}$ Since $\mathrm{Zn}$ is known to induce localized moments and reduce superconducting region, the LSCO system seems to be in similar situation as Zn-doped YBCO. Therefore, we speculate that the different 


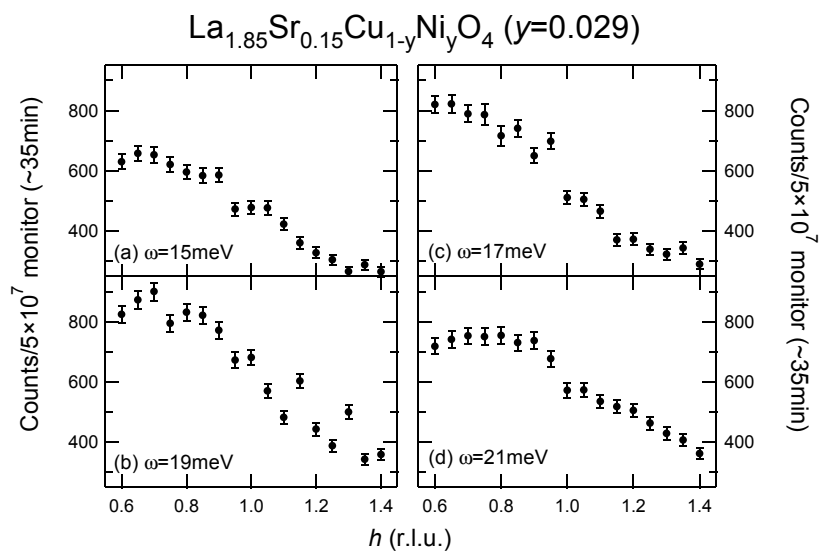

Fig. 4. Profiles of $l$-averaged constant-energy scans for Ni: $y=0.029$ measured at $T=300 \mathrm{~K}$. The scans are same as those in Fig. 3.

temperature dependence of the magnetic peak between LSCO and YBCO is associated with the spacial inhomogeneity of $\mathrm{CuO}_{2}$ plane in the LSCO system.

If the resonance peak and the crossing point peak come from the same origin, the next question is "what determines $E_{\text {cross }}$ and $E_{\text {res }}$ ?" Several theoretical ${ }^{20,21}$ and experimental 22,23 studies claimed that $E_{\text {cross }}$ in the LSCO system depends on $\delta$ assuming the same downwarddispersion. In this view, the crossing point corresponds to the top of the downward dispersion. This model explains large $E_{\text {cross }} \sim 55 \mathrm{meV}$ of the stripe ordered $\mathrm{La}_{1.875} \mathrm{Ba}_{0.125} \mathrm{CuO}_{4}$ by a large $\delta=0.14$. However, the small $E_{\text {cross }}(15 \mathrm{meV})$ for $\mathrm{Ni}: y=0.029$ in spite of almost the same $\delta$ $(\delta \sim 0.12)$ as that of the impurity free sample indicates $\delta$ is not a good parameter to control $E_{\text {cross }}$.

On the other hand, the crossing point could be viewed as the bottom of the the upward dispersion. Tranquada et al. analyzed upward branch observed in stripe ordered LBCO as a spin wave from a two-leg spin ladder. ${ }^{6}$ Barnes et al. have calculated the dispersion of two-leg spin ladder by a $t-J$ model, and have shown that a spingap $\left(E_{\text {gap }}\right)$ is proportional to $J$ and $J_{\perp} / J .{ }^{24}$ Here, $J$ is the magnetic interaction along spin-chain $J$, and $J_{\perp}$ is that between spin-chains. $E_{\text {gap }}$ monotonically increases from 0 with increasing $J_{\perp} / J$, and reaches $J / 2$ at $J=J_{\perp}$. In this model, $E_{\text {cross }}$ can be controlled by changing $J$ and $J_{\perp} / J$. A Cu NQR study on Ni-doped optimum YBCO has shown that $1 / T_{1}$, that is associated with $\chi^{\prime \prime}$, is scaled to $T_{c}$, indicating the energy scale of magnetic excitation, $J$, is scaled by $T_{c}$ for Ni-doping. ${ }^{25}$ Therefore, we speculate that the change in $J$ by Ni-doping affects $E_{\text {cross }}$ and $E_{\text {res }}$ as well as $T_{c}$. Upon Sr-doping in $\mathrm{La}_{2-x} \mathrm{Sr}_{x} \mathrm{CuO}_{4}, J$ is known to decrease from $146 \mathrm{meV}(x=0)^{26}$ to $81 \mathrm{meV}(x=0.16),{ }^{7}$ while $E_{\text {cross }}$ increases linearly with $x$ and reaches $J / 2 \sim 41 \mathrm{meV}$ at $x=0.16 .{ }^{23}$ This might be explained by $J_{\perp}<J$ in the underdoped region and $J_{\perp}=J$ at the optimum doping. 

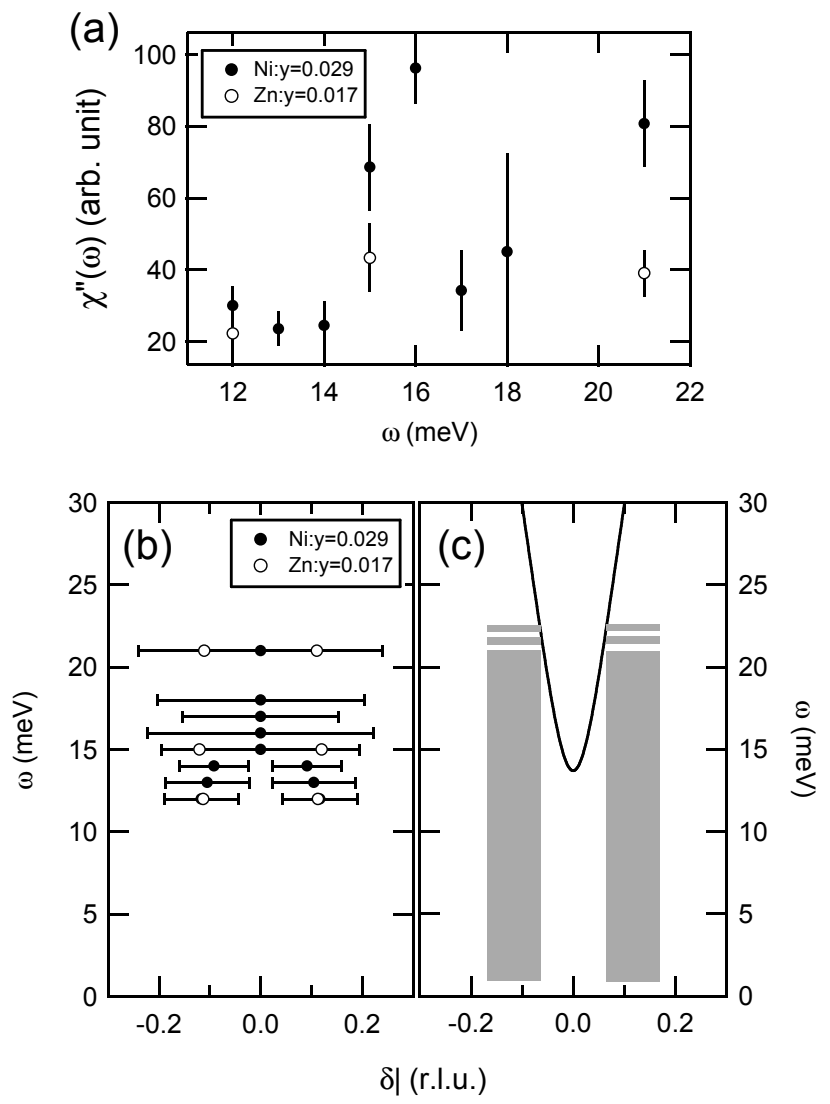

Fig. 5. (a) The $Q$-integrated susceptibility $\chi^{\prime \prime}(\omega)$ and (b) dispersion measured at $T=11 \mathrm{~K}$. The solid and open circles represent the data for Ni: $y=0.029$ and Zn: $y=0.017$, respectively. (c) Schematic diagram of the dispersionless incommensurate fluctuations and spin wave dispersion (described in text).

The solid line in Fig. 5(c) shows a calculated dispersion of the spin-ladder model with $J=27 \mathrm{meV}$. Here, we simulate a spin wave dispersion with one third of $J$ in the impurity free sample with a similar Sr-concentration ${ }^{7}$ assuming both $E_{\text {cross }}$ and $J$ are reduced with the same ratio by Ni-doping. The full-width-at-half maximum (FWHM) of the single peak for $15 \leq \omega \leq 21 \mathrm{meV}$, shown by the horizontal bars in Fig. 5(b), are much broader than the calculated spin wave dispersion. Because incommensurate peaks as sharp as those of the impurity free sample have been observed at $\omega=3 \mathrm{meV}$ in Ni:y $=0.029,{ }^{15}$ the peak-broadening at $\omega=15 \mathrm{meV}$ can not be explained only by impurities. The $Q$-profiles suddenly change from incommensurate peaks to a commensurate peak at $\omega=15 \mathrm{meV}$. Since energy resolutions of these scans are about $1 \mathrm{meV}$, it is unlikely that the incommensurate peaks steeply disperse inward at $\omega=15 \mathrm{meV}$. Instead, the profile above $15 \mathrm{meV}$ is more naturally explained by the sum of incommensurate peaks with the same $\delta$ as that at low energies and the commensurate peak at $(\pi, \pi)$. We thus propose a new view of the magnetic excitations in high- $T_{c}$ cuprates: a crossover from incommensurate peaks to a gapped spin wave excitation shown as a schematic 
diagram in Fig. 5(c).

Vignolle et al. have revealed a two-component structure of magnetic excitations in optimum LSCO from two peaks in $\chi^{\prime \prime}(\omega) .{ }^{7}$ The low energy component, that comes from the incommensurate peaks, has a peak around $18 \mathrm{meV}$ and rapidly decrease above $20 \mathrm{meV}$, whereas the high energy component exhibits a peak near $40 \mathrm{meV}$. If the energy scale of low energy component is renormalized by Ni-doping as high energy component, incommensurate peaks should be attenuated above 6-7 meV. The constant intensity of the incommensurate peaks at least up to $14 \mathrm{meV}$ means that Ni-doping differently renormalizes the energy scales of two components. This suggests different origins for the incommensurate peaks and a gapped spin wave excitation.

Similar incommensurate to a commensurate response has been observed in the paramagnetic state of $\mathrm{Cr}^{27} \mathrm{In} \mathrm{Cr}$, the incommensurate peak shifts inward when a crossover occurs from the coexisting state of the commensurate and incommensurate components to commensurate one at 30-40 meV. We speculate that the downward dispersion below $E_{\text {cross }}$ and $E_{\text {res }}$ observed in the impurity free samples is explained by crossover of two components; incommensurate spin fluctuations and a gapped spin-wave from $(\pi, \pi)$.

In conclusion, we observed that magnetic excitations change from incommensurate to a commensurate response at $E_{\text {cross }}=15 \mathrm{meV}$ for the magnetic impurity-doped $\mathrm{La}_{1.85} \mathrm{Sr}_{0.15} \mathrm{Cu}_{1-y} \mathrm{O}_{4}(\mathrm{Ni}: y=0.029)$. Similar impurity effect on the reduction in $E_{\text {cross }}$ and $E_{\text {res }}$ with a preserved ratio to $T_{c}$ suggests the same origin for the crossing point of hourglasslike dispersion in LSCO and the resonance peak in YBCO. On the other hand, no spectral shift to low energy was observed for the incommensurate peaks, indicating different origins for the two components; incommensurate spin fluctuations at low energies and a dispersive mode at high energy region. We propose a new picture of magnetic excitation in high- $T_{c}$ superconductor; a crossover from incommensurate spin fluctuations to a gapped spin wave rather than the hourglass-like dispersion.

\section{Acknowledgments}

The INS experiments at PONTA was performed under the joint-research program of ISSP, the University of Tokyo. MM was supported by a Grant-In-Aid for Encouragement of Young Scientists (B) (17740217, 2004) from the Japanese Ministry of Education, Science, Sports and Culture. 
J. Phys. Soc. Jpn.

Full PAPER

\section{References}

1) M. Arai, T. Nishijima, Y. Endoh, T. Egami, S. Tajima, K. Tomimoto, Y. Shiohara, M. Takahashi, A. Garrett, and S. M. Bennington: Phys. Rev. Lett. 83 (1999) 608.

2) P. Bourges, Y. Sidis, H. F. Fong, L. P. Regnault, J. Bossy, A. Ivanov, and B. Keimer: Science 288 (2000) 1234.

3) D. Reznik, P. Bourges, L. Pintschovius, Y. Endoh, Y. Sidis, T. Masui, and S. Tajima: Phys. Rev. Lett. 93 (2004) 207003.

4) C. Stock, W. J. L. Buyers, R. A. Cowley, P. S. Clegg, R. Coldea, C. D. Frost, R. Liang, D. Peets, D. Bonn, and W. N. Hardy: Phys. Rev. B 71 (2005) 24522.

5) S. M. Hayden, H. A. Mook, Pengcheng Dai, T. G. Perring and F. Dogan: Nature 429 (2004) 531.

6) J. M. Tranquada, H. Woo, T. G. Perring, H. Goka, G. D. Gu, G. Xu, M. Fujita, and K. Yamada: Nature 429 (2004) 534.

7) B. Vignolle, S. M. Hayden, D. F. McMorrow, H. M. Rønnow, B. Lake, C. D. Frost, and T. G. Perring: Nature Physics 3 (2007) 163.

8) K. Yamada, C. H. Lee, K. Kurahashi, J. Wada, S. Wakimoto, S. Ueki, Y. Kimura, Y. Endoh, S. Hosoya, and G. Shirane: Phys. Rev. B 57 (1998) 6165.

9) P. Dai, H. A. Mook, R. D. Hunt, and F. Doğan: Phys. Rev. B 63 (2001) 54525.

10) J. Rossat-Mignod, L. P. Regnault, C. Vettier, P. Bourges, P. Burlet, J. Bossy, J. Y. Henry, and G. Lapertot: Physica C 185-189 (1991) 86.

11) H. F. Fong, B. Bourges, Y. Sidis, L. P. Regnault, A. Ivanov, G. D. Gu, N. Koshizuka, and B. Keimer: Nature 398 (1999) 588.

12) S. Petit, A. H. Moudden, B. Hennion, A. Vietkin, and A. Revcolevschi: Physica B 234-236 (1997) 800.

13) H. Hiraka, Y. Endoh, M. Fujita, Y. S. Lee, J. Kulda, A. Ivanov, and R. J. Birgeneau: J. Phys. Soc. Jpn 70 (2001) 853.

14) Y. Sidis, P. Bourges, H. F. Fong, B. Keimer, L. P. Regnault, J. Bossy, A. Ivanov, B. Hennion, P. Gautier-Picard, G. Collin, D. L. Millius, and I. Aksay: Phys. Rev. Lett. 84 (2000) 5900.

15) M. Kofu, H. Kimura, and K. Hirota: Phys. Rev. B 72 (2005) 064502.

16) L. Pintschovius, N. Pyka, W. Reichardt, A. Yu. Rumiantsev, N. L. Mitrofanov, A. S. Ivanov, G. Collin, P. Bourges: Physica B 174 (1991) 323.

17) P. Böni, J. D. Axe, G. Shirane, R. J. Birgeneau, D. R. Gabbe, H. P. Jenssen, M. A. Kastner, C. J. Peters, P. J. Picone, and T. R. Thurston: Phys. Rev. B 38 (1988) 185.

18) H. Hiraka, S. Ohta, S. Wakimoto, M. Matsuda, and K. Yamada: J. Phys. Soc. Jpn. 76 (2007) 074703 .

19) Y. Koike, T. Adachi, Y. Tanabe, K. Omori, T. Noji, and H. Sato: J. of Physics 108 (2008) 012003.

20) C. D. Batista, G. Ortiz, and A. V. Balatsky: Phys. Rev. B 64 (2001) 172508.

21) F. Krüger, and S. Scheidl: Phys. Rev. B 67 (2003) 134512.

22) M. Kofu, T. Yokoo, F. Trouw, and K. Yamada: cond-mat/0710.5766.

23) M. Matsuda, M. Fujita, S. Wakimoto, J. A. Fernandez-Baca, J. M. Tranquada, K. Yamada: Phys. Rev. Lett. 101 (2008) 197001.

24) T. Barnes, E. Dagotto, J. Riera, E. S. Swanson: Phys. Rev. B 47 (1993) 3196.

25) Y. Tokunaga, K. Ishida, Y. Kitaoka, and K. Asayama: Solid State Commun. 103 (1997) 43. 
26) R. Coldea, S. M. Hayden, G. Aeppli, T. G. Perring, C. D. Frost, T. E. Mason, S. W. Cheong, and Z. Fisk: Phys. Rev. Lett. 86 (2001) 5377.

27) T. Fukuda, Y. Endoh, K. Yamada, M. Takeda, S. Itoh, M. Arai, and T. Otomo: J. Phys. Soc. Jpn. 65 (1996) 1418. 